\title{
The normal configuration and interindividual differences in intramural lymphatic vessels of the esophagus
}

\author{
Shinji Yajin, MD, ${ }^{\mathrm{a}}$ Gen Murakami, MD, PhD, ${ }^{\mathrm{b}}$ Hiromi Takeuchi, MD, PhD, ${ }^{\mathrm{a}}$ Tadashi Hasegawa, MD, PhD, ${ }^{\mathrm{c}}$ and \\ Hiroya Kitano, $\mathrm{MD}, \mathrm{PhD}^{\mathrm{a}}$
}

\begin{abstract}
Objective: Recently, interindividual differences in lymphatic vessel density among patients with cancer have become a focus of interest for surgeons as a significant prognostic factor. Little morphometric information is available about esophageal lymphatics in the absence of esophageal pathologic changes. We used D2-40 immunohistochemical examination to clarify the normal configuration of the esophageal intramural lymphatics and to evaluate morphometrically preexisting mucosal vessels.
\end{abstract}

\begin{abstract}
Methods: D2-40 immunohistochemical staining for human lymphatic epithelium was performed at three sites in the cervical and thoracic esophagus in 16 donated cadavers without macroscopic malignancy or chronic inflammation. We determined the total numbers of lymphatic vessels and their complete circumferential lengths.

Results: Our immunohistochemical evaluation consistently demonstrated mucosal longitudinal and intermuscular circumferential vessels. In contrast to a previous diagram, the submucosal lymphatics were limited to a few circumferential and marginal vessels. On the basis of these findings, we suggest a new scheme for the intramural lymphatic vessels. In this morphometric study, interindividual differences in number and circumferential length of mucosal lymphatic vessels ranged from $100 \%$ to $200 \%$ (2-3 times), and site-dependent differences were not evident. After correction for esophageal thickness, interindividual differences in lymphatic vessel density were still 2-fold.
\end{abstract}

Conclusion: We hypothesized that the greater length and number of lymphatic vessels in the lamina propria mucosae are likely to provide greater opportunity for lymphatic vessel invasion of cancer. Thus propensity toward cancer invasion would seem to depend not only on cancer pathology but also on individual anatomic features.

Lymphatic vessel invasion has been shown to be a strong independent prognostic factor in cases of esophageal squamous cell cancer. ${ }^{1-3}$ In contrast to identification of lymphatic invasion of cancer with conventional staining, recently developed antibody D2-40 (anti-human podoplanin antibody) techniques have allowed more accurate identification of gastrointestinal mucosal lymphatic vessels, including those of the esophagus. ${ }^{4}$ In this context, many research groups have focused on interindividual differences in lymphatic vessel density among patients with esophageal squamous cell carcinoma. ${ }^{5-7}$ These studies have revealed lymphatic density to be correlated significantly with nodal involvement and thus possibly with prognosis. To our knowledge, however, no previous study has investigated whether certain interindividual differences in lymphatic vessel morphology are present before the onset of cancer pathologic changes.

From the Department of Otorhinolaryngology and Head and Neck Surgery, ${ }^{\mathrm{a}}$ Tottori University School of Medicine, Yonago, Japan; the Division of Internal Medicine and Rehabilitation, ${ }^{\text {b }}$ Iwamizawa Kojin-kai Hospital, Iwamizawa, Japan; and the Department of Clinical Pathology, ${ }^{\mathrm{C}}$ Sapporo Medical University School of Medicine, Sapporo, Japan.

Received for publication Jan 9, 2008; revisions received June 11, 2008; accepted for publication Aug 5, 2008.

Address for reprints: Hiromi Takeuchi, MD, PhD, Tottori University School of Medicine, Otorhinolaryngology and Head and Neck Surgery, Yonago, Tottori, 683-8504 Japan (E-mail: oto3175@grape.med.tottori-u.ac.jp).

J Thorac Cardiovasc Surg 2009;137:1406-14

$0022-5223 / \$ 36.00$

Copyright (c) 2009 by The American Association for Thoracic Surgery doi:10.1016/j.jtcvs.2008.08.069
On the basis of our previous studies on the lymphatics of the esophagus, ${ }^{8-11}$ we hypothesized that significant interindividual differences in the density, number, or length of esophageal intramural lymphatic vessels would be present even in cadavers with supposedly normal anatomy, without malignancy or inflammation. The preexisting anatomic differences in lymphatic vessels would be likely to correlate with the incidence and extent of lymphatic invasion of cancers, perhaps rather than pathologic influences of cancer itself on the lymphatics. On the other hand, the excellent diagram of the intramural esophageal lymphatic system presented by Rice and colleagues ${ }^{12}$ still seems relevant in the recent era of immunohistochemical identification of lymphatics. Consequently, to discuss the anatomic background of cancer metastasis along the lymphatics, we conducted this study (1) to clarify the normal configuration of the intramural esophageal lymphatics by means of D2-40 immunohistochemical examination and (2) to examine morphometrically interindividual and site-dependent variations.

\section{MATERIALS AND METHODS}

We removed specimens of the thoracic esophagus from 30 cadavers (12 male and 18 female) that had been donated to Sapporo Medical University for purposes of anatomic research. These cadavers had been fixed by arterial injection of nonneutralized $10 \%$ formalin solution in water 24 to 48 hours after death. The cadavers ranged in age from 65 to 93 years (mean 82 years). To choose the specimens suitable for this study, we eliminated cadavers in which (1) macroscopic malignancy was present in any cervical, thoracoabdominal or pelvic viscera (5 specimens); (2) leukemia or other blood 
diseases were suspected from blood histologic study of the esophagus (2 specimens); (3) chronic inflammation (mononuclear cell infiltration around ducts of the esophageal glands) was evident, although DeNardi and Ridell ${ }^{13}$ consider this a normal finding (3 specimens); or 4) Barrett esophagus was evident macroscopically, exceeding a craniocaudal length of $5 \mathrm{~cm}$, even if it was unrelated to sites examined for the study (4 specimens). After elimination of the 14 cadavers meeting exclusion criteria, leaving 16 of the 30 cadavers to be examined.

After routine procedures for paraffin embedding of histologic specimens and before immunohistochemical staining, we cut and observed at least 20 serial cross-sections with hematoxylin and eosin staining ( $6 \mu \mathrm{m}$ thick, 120 $\mu \mathrm{m}$ in length along long axis of the esophagus). This was done to minimize potential sampling error in which the circumferential vessel was missed between semiserial cross sections. On the basis of observations of hematoxylin and eosin-stained sections, we eliminated some specimens according to our exclusion criteria to yield our final total of 16 cadavers, as previously stated. After interpreting the histologic study, several semiserial cross-sections (6 $\mu \mathrm{m}$ thick) were prepared at each of the following three sites: (1) the midthoracic level immediately inferior to the tracheal bifurcation, (2) the upper thoracic level close to the lower margin of the aortic arch, and (3) the cervical level immediately inferior to the cricoid cartilage. These sections were stained with hematoxylin and eosin or immunohistochemically for D2-40.

For D2-40 immunohistochemical staining, the primary antibody (1:100 dilution of monoclonal anti-human podoplanin, D2-40 code No. 413451; Nichirei, Tokyo, Japan) was used after immersion in a ligand activator (Histofine SAB-PO Kit, code No. 415211, Nichirei) with autoclave treatment $\left(105^{\circ} \mathrm{C}\right.$ for 10 minutes). The second antibody (Dako Chem Mate Envision Kit; Dako A/S, Glostrup, Denmark) was labeled with horseradish peroxidase, and antigen-antibody reactions were detected by the horseradish peroxidase-catalyzed reaction with diaminobenzidine. Counterstaining with hematoxylin was performed on the same samples. Because immunohistochemical processing was often found to produce uneven staining, we strictly chose good sections from the 16 specimens used for morphometric analysis. As a result of this strict selection process, however, none of the individual esophageal specimens were evaluable at all three sites, and only two of the three sites were available for study. Thus comparisons among all three sites from a single cadaver were not possible in any of the cases.

By means of cross-sectional immunohistochemical histologic staining with D2-40, three of the authors (S.Y., G.M. and T.H.) measured (1) the total number of mucosal lymphatic vessels per section, (2) the numeric density or number of mucosal lymphatic vessels in randomly chosen visual fields with a $\times 40$ objective lens (6-12 fields, depending on size of cross-section), (3) lymphatic vessel density in a hot spot (a field showing highest vessel density) according to the technique of Weidner and associates, ${ }^{14}$ (4) total circumferential length of vessels per section parallel with the total surface area of the lymphatic epithelium, and (5) circumferential length of the basement membrane of the esophageal squamous epithelium per section parallel with the circumferential length of the lumen. Parameters 1 through 3 (numbers of vessels) were measured in at least two sections for one site by three of the authors, and the intermediate result (a value not corresponding to the maximum or minimum) was chosen. When the difference between investigators' counts was too large, the measurement was performed again by case. For estimation of parameters 4 through 5 (lengths), we produced montage photographs of the sections and traced the lymphatic vessels as shown in Figure 1. The lengths were calculated on a Windows-based personal computer with the publicdomain National Institutes of Health Image program (developed at the U.S. National Institutes of Health and available on the internet at http://rsb. info.nih.gov/nih-image/).

The donated cadavers used in this study had been treated post mortem by one of the authors (G.M.) when he was professor and head of the Department of Anatomy at Sapporo Medical University. Written permission had been acquired from each of the donors personally and from their families for use in anatomic research. This study was approved by the ethics committee of our institution. It was performed in accordance with the provisions of the Declaration of Helsinki in 1995 (as revised in Edinburgh in 2000).

\section{RESULTS \\ Histologic Observations of the Intramural Lymphatics}

We observed with immunohistochemical techniques more than 300 of the sections that had been prepared from the 16 cadaveric esophageal specimens. Abundant mucosal lymphatic vessels were consistently observed in the lamina propria mucosae between the squamous epithelium and muscularis mucosae at all three sites and in all the specimens (Figure 1). Because most of the vessels were cut transversely or obliquely, they appeared to run longitudinally along most of the long axis of the esophagus, rather than circumferentially or transversely. In general, the lymphatic vessel density was high in mucosal folds protruding into the lumen, whereas it was low at the concavity or bottom of the folds. There was no evidence of any site-dependent difference or laterality in density, however, such as ventral quarter dominancy. Although efferent vessels of the mucosal lymphatics were difficult to find, the rare examples encountered were connected to a circumferential vessel running along the outer margin of the tela submucosa (Figure 2, A). Thus the tela submucosa (a layer between the muscularis mucosae and muscularis propria) itself contained few lymphatic vessels, even around the glands and their ducts. The marginal, submucosal lymphatic vessels issued efferent vessels, which usually accompanied an artery, vein, and nerve (Figure 3, A) or sometimes independently penetrated the inner circular muscle of the muscularis propria without a concomitant artery and vein (Figure 3, B).

In the narrow intermuscular space of the muscularis propria containing the artery, vein, and nerve plexus of Auerbach, we often observed a lymphatic plexus made up of circumferentially running lymphatic vessels (Figures 2 and 3). Sometimes at the upper thoracic level, however, and often at the cervical level, the space became unclear because the outer longitudinal muscles were intermingled with the inner circular layer (Figure 2, C). Thus, conversely, the intermuscular lymphatic plexus was most evident at the midthoracic level among the three sites examined. Afferent vessels of the intermuscular plexus usually ran along the artery and vein and connected with the lymphatics embedded in the adventitial tissue around the esophagus. Independent lymphatic vessels, however, were sometimes seen passing through the outer muscle layer, exhibiting a tortuous course. During preparation of these specimens, we confirmed macroscopically that they did not contain any direct drainage vessels extending from the lamina propria mucosae to the thoracic duct.

\section{Morphometric Data for the Mucosal Lymphatics}

We measured lymphatic vessels in the lamina propria mucosae in cross-sections of 16 esophageal specimens. 


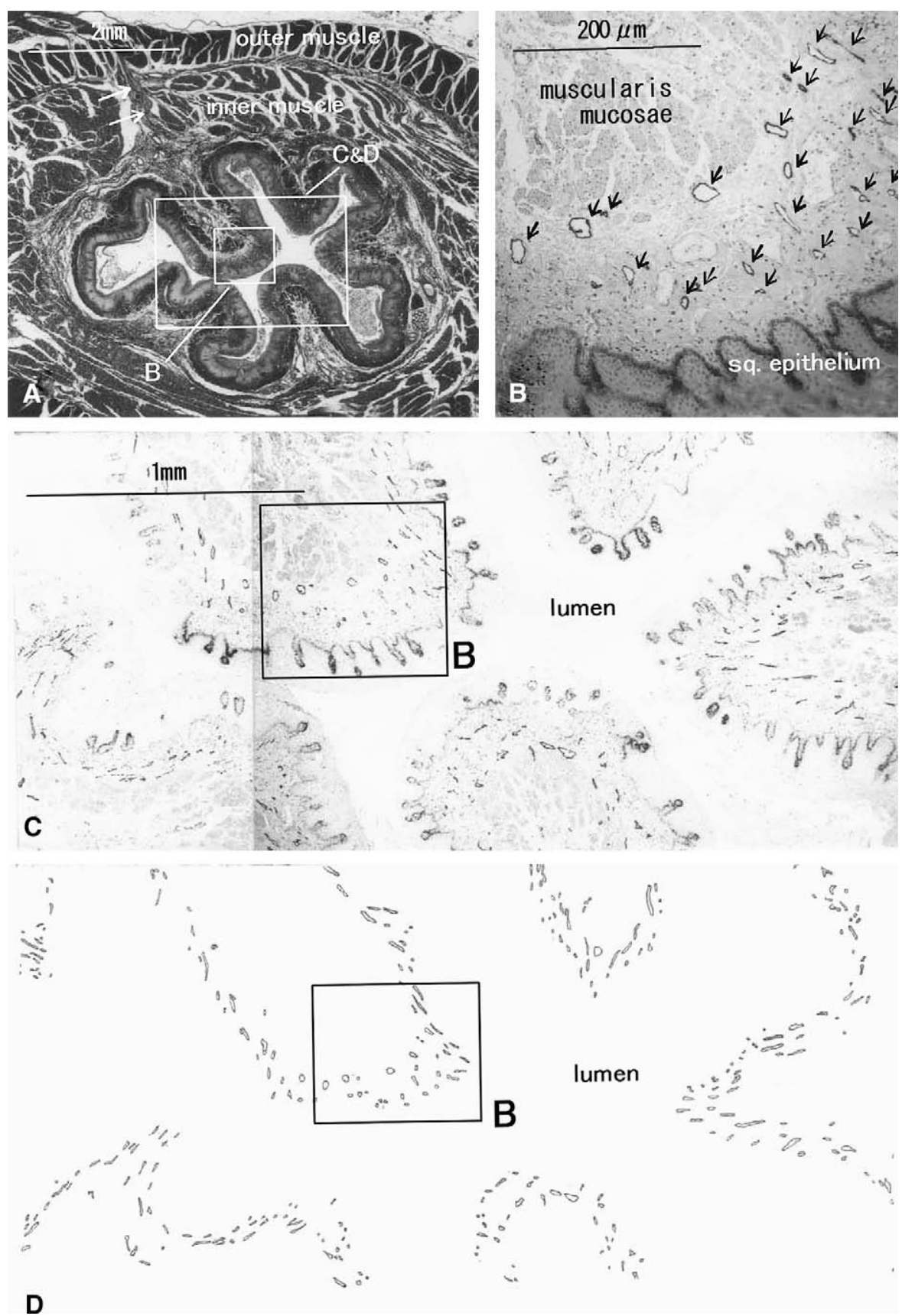

FIGURE 1. Mucosal lymphatic vessel distribution at level immediately inferior to tracheal bifurcation. A, Almost entire horizontal section of esophagus at level of tracheal bifurcation. Upper part corresponds to ventral side of esophagus. Arrows indicate vascular bundle passing through inner circular and outer longitudinal muscle layers (inner muscle, outer muscle). B and C, D2-40 immunohistochemical staining for area indicated by square is shown. B, Highermagnification view displays abundant lymphatic vessels (arrows) in lamina propria mucosae between stratified squamous epithelium and muscularis mucosa. Some vessels are dilated, and others are flattened or collapsed. Squamous epithelium basal layer and lymphatic vessels stain for D2-40. Most mucosal vessels are cut transversely or obliquely. C, Lower-magnification view includes area in B. D, Hand tracing with pen of C. Because of lower magnification, C and D exhibit no difference in mucosal lymphatic vessel density between parts or quarters of section.

The total number of mucosal vessels per section exhibited significant interindividual differences: 179 to 504 (mean 353) lymphatic vessels at the midthoracic level, 185 to 621 (mean 386) at the upper thoracic level, and 226 to
510 (mean 328) at the cervical level. Likewise, the total circumferential length of all vessels per section, which almost paralleled the total surface area of the lymphatic epithelium, also displayed significant interindividual 

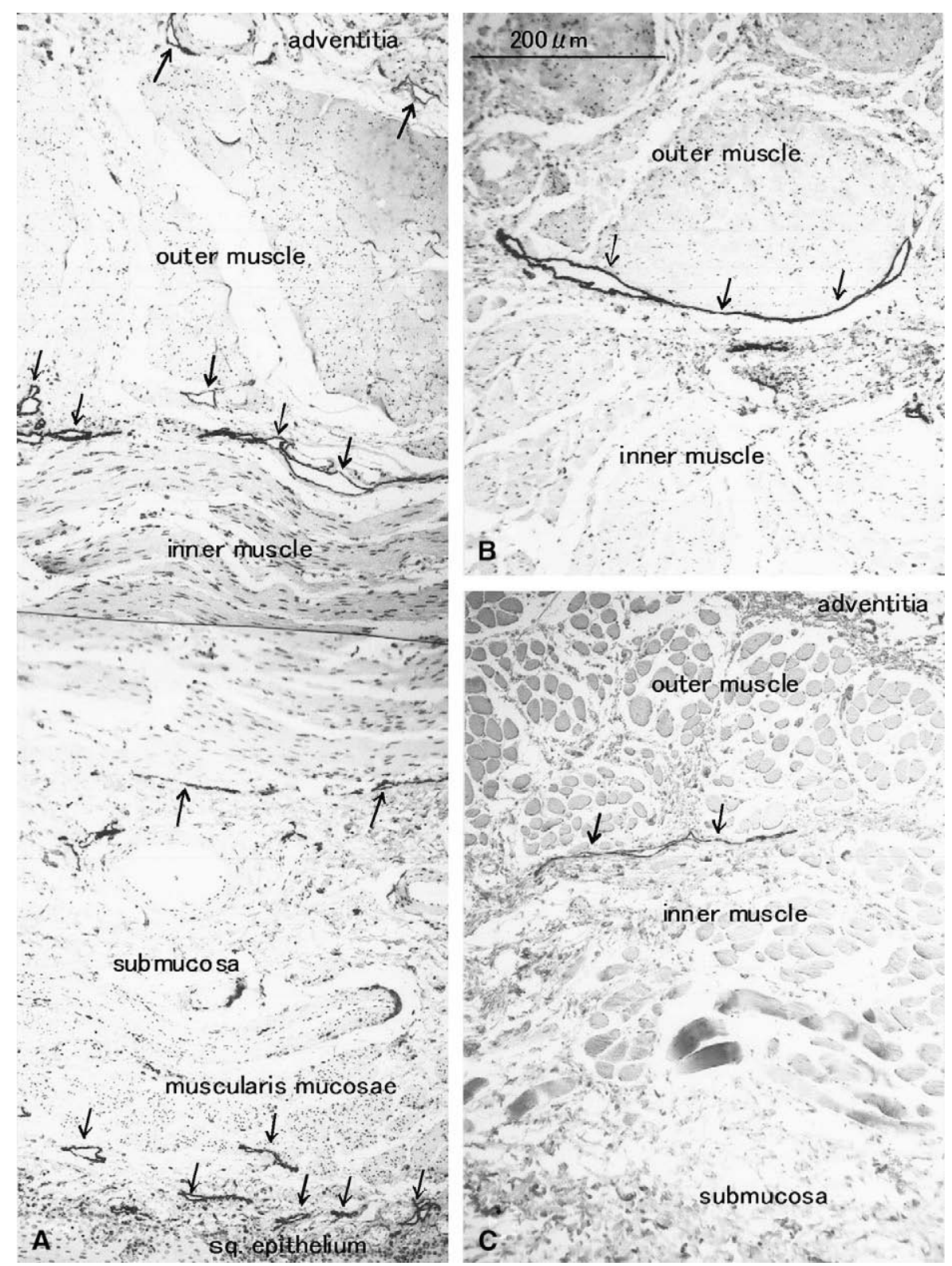

FIGURE 2. Esophageal intramural lymphatics outside mucosal layer. A, Entire esophageal wall (squamous epithelium, muscularis mucosae, tela submucosa, and inner circular and outer longitudinal muscle layers) at level of tracheal bifurcation. Arrows indicate lymphatic vessels staining for D2-40. Lymphatic vessels are evident (1) in lamina propria mucosae, (2) along outer surface of tela submucosa, and (3) between inner and outer muscle layers. Latter two vessels appear to run circumferentially. B and C, Specimens obtained from different cadavers exhibiting lymphatic vessels running between inner and outer muscle layers at level of the aortic arch. Note abundant muscle fibers cut axially in inner circular layer. All panels were photographed at same magnification; bar in B represents $200 \mu \mathrm{m}$.

differences: 22.9 to $44.1 \mathrm{~mm}$ (mean $31.7 \mathrm{~mm}$ ) at the midthoracic level, 10.8 to $43.3 \mathrm{~mm}$ (mean $26.1 \mathrm{~mm}$ ) at the upper thoracic level, and 14.5 to $37.4 \mathrm{~mm}$ (mean $22.7 \mathrm{~mm}$ ) at the cervical level. These data did not exhibit clear differences between sites in any individual specimen. An individual specimen with a higher value at one of the three sites, however, tended to show higher values for vessels at the other site examined.
The number of mucosal lymphatic vessels in a randomly chosen visual field with a $\times 40$ objective lens (numeric density) exhibited interindividual differences, especially at the cervical level. There were 6.9 to 8.9 vessels per field (mean in individual specimens) at the midthoracic level, 5.9 to 8.5 at the upper thoracic level, and 5.6 to 10.7 at the cervical level. When the lymphatic vessels were dilated, the number per visual field was reduced. Among the ventral, 

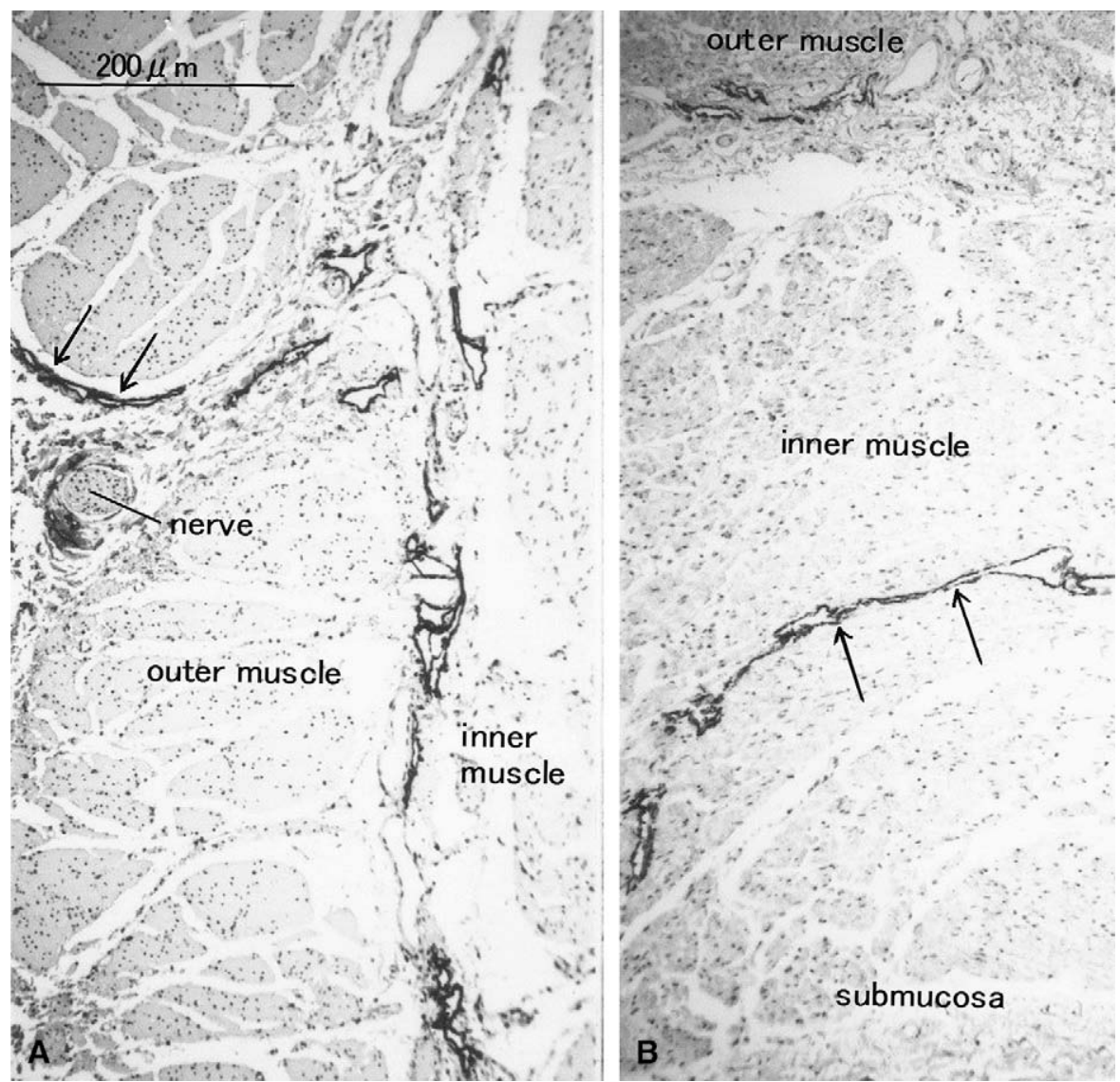

FIGURE 3. Drainage routes of the intermuscular and submucosal vessels. A, Left side corresponds to outer aspect of esophagus and displays drainage vessel (arrows) of intermuscular lymphatics. B, Lymphatic vessel (arrows) passing through inner circular muscle mass and connecting submucosal and intermuscular lymphatic vessels. Both A and B were taken at level of tracheal bifurcation, but from different specimens, and are at same magnification; bar in A represents $200 \mu \mathrm{m}$.

dorsal, left, and right quarters of a cross-section, however, no clear differences in density were evident. The averaged densities at the three sites were similar: 7.7 vessels per field at the midthoracic level, 7.0 vessels at the upper thoracic level, and 7.1 vessels at the cervical level. Likewise, the hot spot method (a type of numeric density estimation in which the targets are counted in a selected field with the highest density), also revealed considerable interindividual differences: 8 to 13 (mean 11.3) lymphatic vessels at the midthoracic level, 8 to 12 (mean 9.8) at the upper thoracic level, and 9 to 15 (mean 11.3) at the cervical level (Figure 4). The cervical esophagus tended to display greater interindividual differences than did the thoracic esophagus.

The length of the basement membrane of the esophageal squamous epithelium per section, which ran in parallel with the circumferential length of the lumen, ranged from 27.5 to $48.9 \mathrm{~mm}$ (mean $37.6 \mathrm{~mm}$ ) at the midthoracic level, 16.4 to $44.6 \mathrm{~mm}$ (mean $31.54 \mathrm{~mm}$ ) at the upper thoracic level, and 14.5 to $37.4 \mathrm{~mm}$ (mean $22.7 \mathrm{~mm}$ ) at the cervical level. Thus the mucosal epithelium per section tended to be longer at the lower level of the esophagus. Interindividual differences were so evident, however, with the upper thoracic and cervical levels showing a difference of more than 2 -fold (or 100\%), that they were likely to mask any site-dependent differences in the mucosal lymphatics. We therefore addressed the issue of whether thicker esophageal specimens simply contained a greater length or number of mucosal lymphatic vessels.

Because of the great differences in thickness of the esophagus or in the circumferential length of the lumen, we determined the length of mucosal lymphatics per 1-mm length of the epithelium (the total vascular length divided by the epithelial length), and the values obtained were as follows: 0.64 to $1.11 \mathrm{~mm}$ (mean $0.85 \mathrm{~mm}$ ) at the midthoracic level, 0.65 to $1.06 \mathrm{~mm}$ (mean $0.81 \mathrm{~mm}$ ) at the upper thoracic level, and 0.60 to $1.16 \mathrm{~mm}$ (mean $0.72 \mathrm{~mm}$ ) at the cervical level (Figure 4). Likewise, the number of mucosal lymphatic vessels per section was divided by the circumferential epithelial length to provide the lymphatic vessel density per 1-mm length of the epithelium, and these values usually ranged from 8 to 13 vessels per 1-mm length of the esophageal epithelium (Figure 4). Even after such corrections, however, 

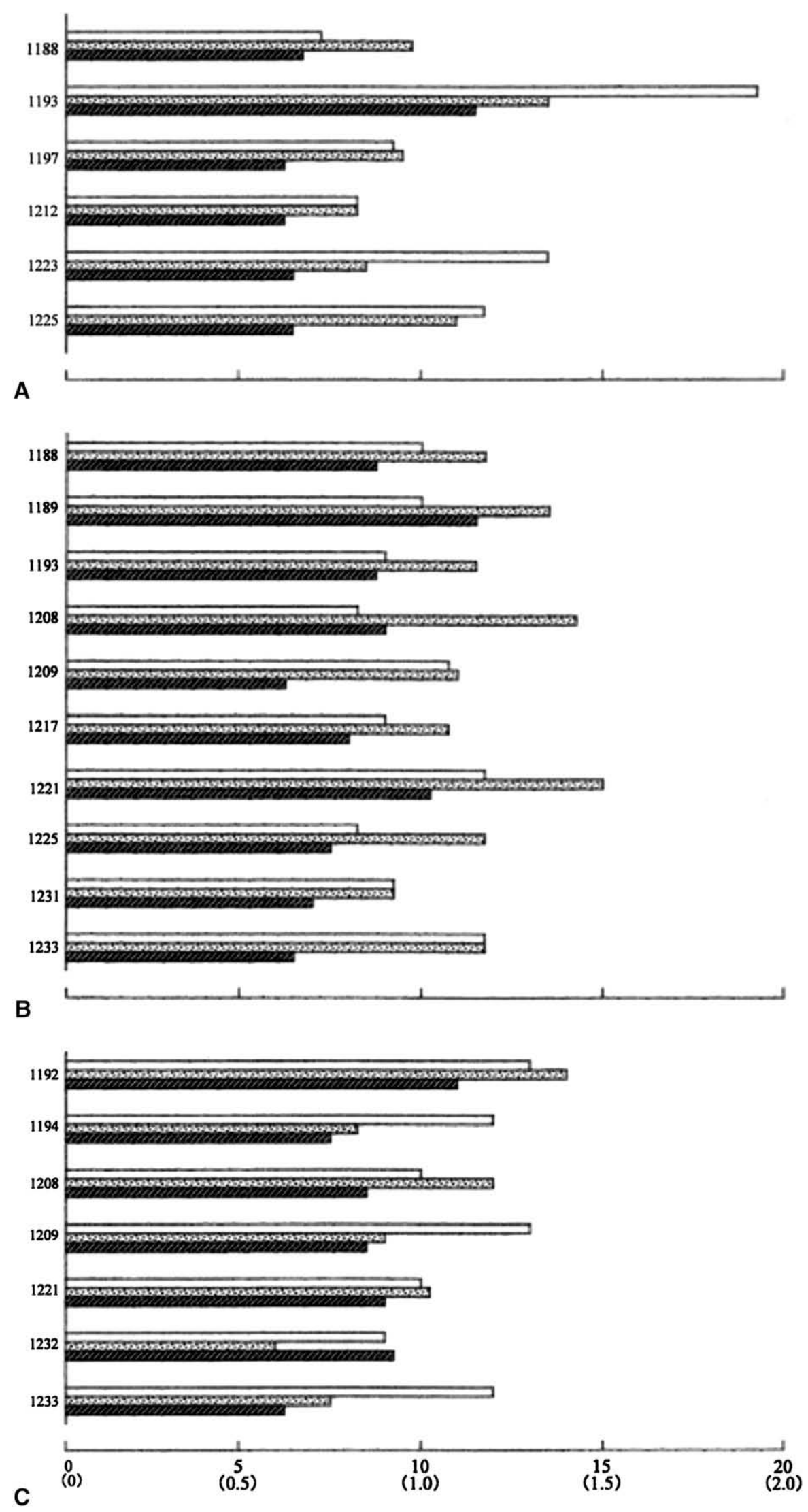
some individual specimens showed obviously longer or more numerous mucosal lymphatic vessels, as for example specimens 1223 at the cervical level 1208 and 1221 at the upper thoracic level, and 1233 at the midthoracic level (Figure 4). These large values for length or number were almost double the smallest value. In these specimens showing the highest values, we again confirmed that there was no evidence of chronic inflammation such as mononuclear cell infiltration along the ducts of the esophageal glands.

\section{DISCUSSION}

With D2-40 immunohistochemical staining, we clearly demonstrated that mucosal lymphatic vessels were densely distributed in the lamina propria mucosae of the esophagus. Observation of a limited area with a $\times 20$ or $\times 40$ objective lens revealed that the distribution was uneven. Most of the mucosal lymphatic vessels ran longitudinally and were limited in distribution circumferentially, which was essentially consistent with our previous work on specific specimens showing vessels draining directly from the mucosa to the thoracic duct ${ }^{9}$ as well as with the classic description by Weinberg. ${ }^{15}$ The intramuscular lymphatic vessels were also often clearly observed, especially in the intermuscular space for the nerve plexus of Auerbach. In contrast to the mucosal vessels, the intermuscular vessels usually ran circumferentially. Sometimes, lymphatic vessels independently penetrated the muscularis propria (either the outer or the inner layer) without a concomitant artery and vein. We were unable, however, to obtain pictures of lymphatic vessels passing through the muscularis mucosae.

Unexpectedly, we rarely observed lymphatic vessels in the tela submucosa, except for vessels running circumferentially along the outer margin of this layer. Thus presumably the number and density of submucosal vessels were likely to be much lower, even around glandular tissue, than those of mucosal and intramuscular lymphatic vessels. This observation clearly differed from the well-known diagram presented by Rice and colleagues. ${ }^{12}$ In combination with our previous observation, ${ }^{9}$ we therefore postulate a new scheme for the esophageal intramural lymphatics (Figure 5). Although superficially this scheme resembles that postulated by Rice and colleagues, ${ }^{12}$ the lymphatic morphology is quite different from the latter; circumferential vessel dominancy changes to longitudinal vessel dominancy, depending on the laminations of the esophageal wall. Sentinel node navigation surgery would seem to be disturbed by the innermost layer, because the abundant longitudinal mucosal vessels are likely to mislead multiple sentinel nodes. Further study with surgical specimens will be necessary to clarify the submucosal lymphatic morphology in view of the poorly developed lymphatic vessels noted in the tela submucosa in this investigation. We speculate that the submucosal lymphatics would undergo a drastic change in their normal configuration under the influence of cancer.

In this work, we counted and measured all D2-40-positive lymphatic vessels in the lamina propria mucosae per section. This method is in contrast to the usual hot spot method, ${ }^{14}$ in which targets are counted in a selected field showing the maximum number. Notably, the actual number and circumferential length of the vessels displayed interindividual differences of 2 - to 3 -fold $(100 \%-200 \%)$, rather than small site-dependent differences. Strictly speaking, the latter differences seemed to be masked by the former. The observed variations in the thickness of the esophagus, however, exceeded our expectation. Thus the circumferential length of the epithelial basement membrane also displayed a difference of more than 2 -fold, or $100 \%$. The primary step of cancer metastasis would occur along the basement membrane of the stratified epithelium. We therefore determined the length of the mucosal lymphatics per 1-mm length of the epithelium and the lymphatic vessel density per 1-mm length of the epithelium (Figure 4). Notably, after such corrections, interindividual differences were still evident: the greatest values exceeded the smallest by 2 -fold or more, although in specimens with the highest values, we did not find any evidence of chronic inflammation. Thus the "preexisting" mucosal lymphatic vessels also seemed to exhibit significant interindividual differences.

With the hot spot method, Brundler and coworkers, ${ }^{7} \mathrm{Na}-$ kayama and colleagues, ${ }^{6}$ and Mori and colleagues ${ }^{5}$ determined mucosal lymphatic vessel density in nonpathologic areas of surgically removed esophageal specimens (Table 1). Our counting method was the same as that used by Brundler and coworkers. ${ }^{7}$ When we tried to read the values in their graphic representation, their value of $9.1 \pm 0.3$ vessels per objective field was similar to ours. although they measured the lower esophagus. Our current data, however, showed a greater SD or interindividual difference. In contrast, the other two Japanese groups seemed to use a modification of the original hot spot method, making comparison with our data difficult. Notably, however, these data also displayed very large SDs (Table 1). We speculate that the range of interindividual

FIGURE 4. Graphic demonstrations of interindividual differences in lymphatic vessel density and vascular length. Data for cervical (A), upper thoracic (B), and midthoracic (C) esophagus. White bars represent numeric density according to hot spot method (greatest number of vessels in selected visual field with $\times 40$ objective lens). Dotted bars represent numeric density per 1-mm epithelium (numbers of all lymphatic vessels divided by circumferential length of epithelial basement membrane). Black bars represent length of mucosal lymphatics per 1-mm length of epithelium (epithelial length of all lymphatic vessels divided by circumferential length of epithelial basement membrane). Axes: x axis indicates (1) two types of numeric density with scales of 0 , 5 , 10, 15, and 20, and (2) lymphatic vascular length (mm) with scales in parentheses of $0,0.5,1.0,1.5$, and 2.0; y axis indicates specimen numbers. 


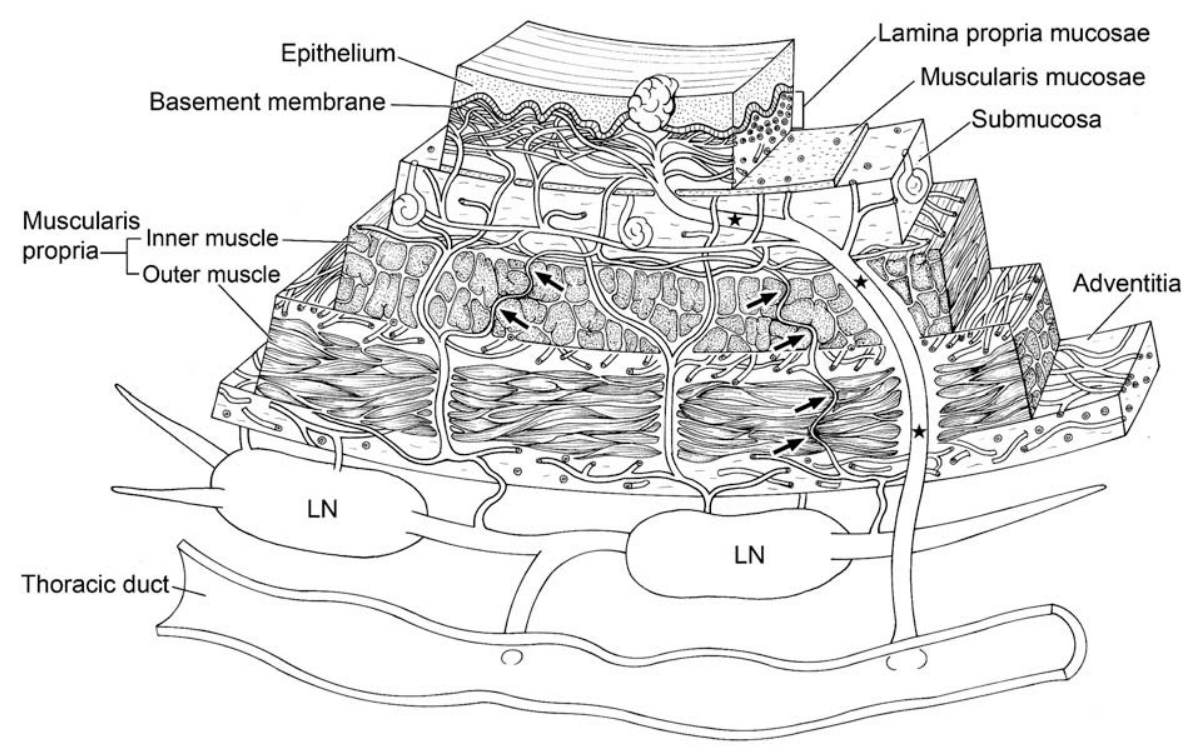

FIGURE 5. Schematic representation of intramural lymphatics of thoracic esophagus. Lamina propria mucosae contains abundant longitudinally running lymphatic vessels, whereas circumferentially running vessels are dominant in intermuscular space between inner and outer muscle layers of muscularis propria, as well as along outer margin of tela submucosa. Efferent vessels usually accompany artery and vein (artery and vein not shown) but sometimes penetrate muscularis propria (arrowheads). Thick direct drainage vessel from mucosal lymphatics (stars) passes along large gap in muscularis propria. $L N$, Lymph node.

differences differs among human populations. In addition to the hot spot method, we also measured the numbers of lymphatic vessels in a $\times 40$ visual field selected at random (6-12 fields, depending on thickness of the esophagus). This is the usual method for obtaining the numeric density of a histologic structure. ${ }^{16}$ The smallest value in these 6 to 12 fields usually corresponded to half the value in a hot spot. The hot spot method is conventional and seems to be good for identification of a local response of host lymphatics to cancer. Because of the narrow visual field, however, the hot spot does not seem to reflect the normal morphology of the mucosal lymphatics, including the uneven distribution of the lymphatic vessels. For instance, in specimens 1193 and 1223 at the cervical level and 1192 and 1232 at the midthoracic level (Figure 4), the hot spot method might have overestimated the lymphatic vessel density.

Some recent studies have demonstrated paradoxic suppression of lymphangiogenesis in breast cancer $^{17-19}$ and esophageal adenocarcinoma. ${ }^{7}$ It seems easy, however, to hypothesize that longer or more numerous lymphatic vessels are likely to provide a greater opportunity for (and thus higher incidence of) mucosal lymphatic vessel invasion by cancer. Whether cancer invasion is easy or difficult seems to depend on individual anatomy as well as pathologic and cytologic characteristics. In particular, our study revealed an interindividual difference in lymph vessel density of almost 2-fold. A background difference in basic or physiologic vascular endothelial growth factor $\mathrm{C}$ expression appeared to be present. If so, the fact that the finding of vascular endothelial growth factor $\mathrm{C}$ messenger RNA is positive in fewer than $50 \%$ of patients with esophageal squamous cell carcinoma ${ }^{20-22}$ seems to correspond to this anatomic difference. Nevertheless, despite the densely packed mucosal vessels, it is well known that intramucosal esophageal cancer rarely produces nodal metastasis. In our study, a lymph drainage route was less frequently found crossing the tela submucosa and inner muscular layer to connect with the intramuscular vessels. Moreover, in our previous work, ${ }^{8,9,11}$ the direct drainage route from the mucosal lymphatics was seen to be located in the limited area. We therefore speculate that mucosal lymphatic invasion of cancer often requires a relatively long lag time before reaching the regional node.

In summary, this immunohistochemical study revealed the following in the adult esophagus: (1) the mucosal

TABLE 1. Lymphatic vessel densities measured by hot spot method in nonpathologic esophageal mucosa according to previous reports and current study

\begin{tabular}{llcc}
\hline \multicolumn{1}{c}{ Study } & Site & Magnification & $\begin{array}{c}\text { Lymphatic density } \\
\text { (vessels/field) }\end{array}$ \\
\hline${\text { Brundler et } \mathrm{al}^{7}}$ & Lower & $\times 400$ & $9.1 \pm 0.3$ \\
Nakayama et al $^{6}$ & NS & $\times 200$ & $7.2 \pm 3.3$ (average, 3 \\
& & & fields)* \\
Mori et al $^{5}$ & NS & $\times 200$ & $16.5 \pm 12.1$ (sum, 2 \\
& & & fields)* \\
Current study & Middle & $\times 400$ & $11.3 \pm 1.6$ \\
& Upper & $\times 400$ & $9.8 \pm 1.5$ \\
\hline
\end{tabular}

NS, Not specified (authors did not describe sites examined). *Researchers do not appear to have counted vessels in the entire objective field but rather in a grid used for taking photographs. 
longitudinal lymphatic vessels were much more dominant than the circumferential submucosal and intermuscular vessels at any sites, and (2) the mucosal lymphatic vessel density displayed an almost 2-fold interindividual difference after correction according to differences in thickness (size) of the esophagus. We simply believe that, for the primary step of cancer metastasis, the frequency is greater in a patient with 200 preexisting mucosal vessels per unit than in one with 100. In contrast to our measurement of actual vascular lengths or numbers, the usual hot spot method seems to be limited for evaluation of individual difference.

\section{References}

1. Brücher BL, Stein HJ, Werner M, Siewert JR. Lymphatic vessel invasion is an independent prognostic factor in patients with a primary resected tumor with esophageal squamous cell carcinoma. Cancer. 2001;92:2228-33.

2. Watanabe M, Kuwano H, Araki K, Kawaguchi H, Saeki H, Kitamura K, et al. Prognostic factors in patients with submucosal carcinoma of the oesophagus. Br J Cancer. 2000;83:609-13.

3. Torres CM, Wang HH, Turner JR, Richards W, Sugarbaker D, Shahsafaei A, et al. Pathologic prognostic factors in oesophageal squamous cell carcinoma: a followup study of 74 patients with or without preoperative chemoradiation therapy. Mod Pathol. 1999;12:961-8.

4. Yonemura Y, Endou Y, Tabachi K, Kawamura T, Yun HY, Kameya T, et al. Evaluation of lymphatic invasion in primary gastric cancer by a new antibody, D2-40. Human Pathol. 2006;37:1193-9.

5. Mori D, Yamasaki F, Shibata M, Tokunaga O. Lateral peritumoral lymphatic vessel invasion can predict lymph node metastasis in esophageal squamous cell carcinoma. Mod Pathol. 2007;20:694-700.

6. Nakayama Y, Matsumoto K, Nagato M, Inoue Y, Katsuki T, Minagawa N, et al. Significance of lymphangiogenesis as assessed by immunohistochemistry for podoplanin in patients with esophageal carcinoma. Anticancer Res. 2007;27:619-25.

7. Brundler MA, Harrison JA, deSaussure B, dePerrot M, Pepper MS. Lymphatic vessel density in the neoplastic progression of Barrett's oesophagus to adenocarcinoma. J Clin Pathol. 2006;59:191-5.

8. Murakami G, Sato I, Shimada K, Dong C, Kato Y, Imazeki T. Direct lymphatic drainage of the esophagus to the thoracic duct. Surg Radiol Anat. 1994;16:399-407.
9. Kuge K, Murakami G, Mizobuchi S, Hata Y, Aikou T, Sasaguri S. Submucosal territory of the direct lymphatic drainage system to the thoracic duct in the human esophagus. J Thorac Cardiovasc Surg. 2003;125:1343-9.

10. Mizutani M, Nawata S, Hirai I, Murakami G, Kimura W. Anatomy and histology of Virchow's node. Anat Sci Int. 2005;80:194-9.

11. Mizutani M, Murakami G, Nawata S, Hirai I, Kimura W. Anatomy of right recurrent nerve node: why does early metastasis of esophageal cancer occur in it? Surg Radiol Anat. 2006;28:333-8.

12. Rice TW, Zuccaro G Jr., Adelstein DJ, Rybicki LA, Blackstone EH, Goldblum JR. Esophageal carcinoma: depth of tumor invasion is predictive of regional lymph node status. Ann Thorac Surg. 1998;65:787-92.

13. DeNardi FG, Ridell RH. Esophagus. In: Sternberg SS, ed. Histology for pathologists. Philadelphia: Lippincott-Raven; 1997:461-80.

14. Weidner N, Semple JP, Welch WR, Folkman J. Tumor angiogenesis and metastasis-correlation in invasive breast carcinoma. N Engl J Med. 1991;324: 1-8.

15. Weinberg JA. Lymphatics of the esophagus. In: Haagensen CD, Feind CR, Herter FP, Slanetz CA Jr., Weinberg JA, eds. The lymphatics in cancer. Philadelphia: Saunders; 1972:245-9.

16. Uchiyama Y, Oomiya A, Murakami G. Fluctuations in follicular structures of rat thyroid glands during 24 hours. Am J Anat. 1986;175:23-33.

17. Agarwal B, Saxena R, Moriyama A, Mehrotra S, Badove S. Lymphangiogenesis does not occur in breast cancer. Am J Surg Pathol. 2005;29:1449-55.

18. Choi WW, Lewis MM, Lawson D, Yin-Goen Q, Birdsong GG, Cotsonis GA, et al. Angiogenic and lymphangiogenic microvessel density in breast carcinoma: correlation with clinicopathologic parameters and VEGF-family gene expression. Mod Pathol. 2005; 18:143-52.

19. van der Auwera I, van den Eynden GG, Colpaert CG, van Laere SJ, van Dam P, van Marck EA, et al. Tumor lymphangiogenesis in inflammatory breast carcinoma: a histomorphometric study. Clin Cancer Res. 2005;11:7637-42.

20. Kimura Y, Watanabe M, Ohga T, Saeki H, Kakeji Y, Baba H, et al. Vascular endothelial growth factor $\mathrm{C}$ expression correlates with lymphatic involvement and poor prognosis in patients with esophageal squamous cell carcinoma. Oncol Rep. 2003;10:1747-51.

21. Ding MX, Lin XQ, Fu XY, Zhang N, Li JC. Expression of vascular endothelial growth factor-C and angiogenesis in esophageal squamous cell carcinoma. World J Gastroenterol. 2006;12:4582-5.

22. Matsumoto M, Natsugoe S, Okumura H, Arima H, Yanagita S, Uchikado Y, et al. Overexpression of vascular endothelial growth factor-C correlates with lymph node micrometastasis in submucosal esophageal cancer. $J$ Gastrointest Surg. 2006;10:1016-22. 